\title{
Colligative Properties of Solutions: I. Fixed Concentrations
}

\author{
Kenneth S. Alexander, ${ }^{1}$ Marek Biskup, ${ }^{2}$ and Lincoln Chayes ${ }^{2}$
}

Received July 15, 2004; accepted December 2, 2004

\begin{abstract}
Using the formalism of rigorous statistical mechanics, we study the phenomena of phase separation and freezing-point depression upon freezing of solutions. Specifically, we devise an Ising-based model of a solvent-solute system and show that, in the ensemble with a fixed amount of solute, a macroscopic phase separation occurs in an interval of values of the chemical potential of the solvent. The boundaries of the phase separation domain in the phase diagram are characterized and shown to asymptotically agree with the formulas used in heuristic analyses of freezing-point depression. The limit of infinitesimal concentrations is described in a subsequent paper.
\end{abstract}

KEY WORDS: Freezing-point depression; phase separation; droplet transition; Wulff construction; Ising model; canonical ensemble.

\section{INTRODUCTION}

\subsection{Motivation}

The statistical mechanics of pure systems - most prominently the topic of phase transitions and their associated surface phenomena - has been a subject of fairly intensive research in recent years. Several physical principles for pure systems (the Gibbs phase rule, Wulff construction, etc.) have been put on a mathematically rigorous footing and, if necessary, supplemented with appropriate conditions ensuring their validity. The corresponding phenomena in systems with several mixed components, particularly solutions, have long been well-understood on the level of

\footnotetext{
(C) 2004 by K.S. Alexander, M. Biskup and L. Chayes. Reproduction, by any means, of the entire article for non-commercial purposes is permitted without charge.

${ }^{1}$ Department of Mathematics, USC, Los Angeles, California, USA.

${ }^{2}$ Department of Mathematics, UCLA, Los Angeles, California, USA.
} 
theoretical physics. However, they have not received much mathematically rigorous attention and in particular have not been derived rigorously starting from a local interaction. A natural task is to use the ideas from statistical mechanics of pure systems to develop a higher level of control for phase transitions in solutions. This is especially desirable in light of the important role that basic physics of these systems plays in sciences, both general (chemistry, biology, oceanography) and applied (metallurgy, etc.). See e.g. (refs. 11, 24, 27) for more discussion.

Among the perhaps most interesting aspects of phase transitions in mixed systems is a dramatic phase separation in solutions upon freezing (or boiling). A well-known example from "real world" is the formation of brine pockets in frozen sea water. Here, two important physical phenomena are observed:

(1) Migration of nearly all the salt into whatever portion of ice/water mixture remains liquid.

(2) Clear evidence of facetting at the water-ice boundaries. Quantitative analysis also reveals the following fact:

(3) Salted water freezes at temperatures lower than the freezing point of pure water. This is the phenomenon of freezing-point depression.

Phenomenon (1) is what "drives" the physics of sea ice and is thus largely responsible for the variety of physical effects that have been observed, see e.g. (refs. 17, 18). Notwithstanding, (1-3) are not special to the saltwater system; they are shared by a large class of the so called non-volatile solutions. A discussion concerning the general aspects of freezing/boiling of solutions - often referred to as colligative properties - can be found in refs. 24 and 27.

Of course, on a heuristic level, the above phenomena are far from mysterious. Indeed, (1) follows from the observation that, macroscopically, the liquid phase provides a more hospitable environment for salt than the solid phase. Then (3) results by noting that the migration of salt increases the entropic cost of freezing so the energy-entropy balance forces the transition point to a lower temperature. Finally, concerning observation (2) we note that, due to the crystalline nature of ice, the ice-water surface tension will be anisotropic. Therefore, to describe the shape of brine pockets, a Wulff construction has to be involved with the caveat that here the crystalline phase is on the outside. In summary, what is underlying these phenomena is a phase separation accompanied by the emergence of a crystal shape. In the context of pure systems, such topics have been well understood at the level of theoretical physics for quite some time ${ }^{(12,16,32,33)}$ and, 
recently (as measured on the above time scale), also at the level of rigorous theorems in two $(2,4,14,22,28,29)$ and higher ${ }^{(6,9,10)}$ dimensions.

The purpose of this and a subsequent paper is to study the qualitative nature of phenomena (1-3) using the formalism of equilibrium statistical mechanics. Unfortunately, a microscopically realistic model of salted water/ice system is far beyond reach of rigorous methods. (In fact, even in pure water, the phenomenon of freezing is so complex that crystallization in realistic models has only recently - and only marginally - been exhibited in computer simulations. ${ }^{(26)}$ ) Thus we will resort to a simplified version in which salt and both phases of water are represented by discrete random variables residing at sites of a regular lattice. For these models we show that phase separation dominates a non-trivial region of chemical potentials in the phase diagram - a situation quite unlike the pure system where phase separation can occur only at a single value (namely, the transition value) of the chemical potential. The boundary lines of the phaseseparation region can be explicitly characterized and shown to agree with the approximate solutions of the corresponding problem in the physicalchemistry literature.

The above constitutes the subject of the present paper. In a subsequent paper (1) we will demonstrate that, for infinitesimal salt concentrations scaling appropriately with the size of the system, phase separation may still occur dramatically in the sense that a non-trivial fraction of the system suddenly melts (freezes) to form a pocket (crystal). In these circumstances the amount of salt needed is proportional to the boundary of the system which shows that the onset of freezing-point depression is actually a surface phenomenon. On a qualitative level, most of the aforementioned conclusions should apply to general non-volatile solutions under the conditions when the solvent freezes (or boils). Notwithstanding, throughout this and the subsequent paper we will adopt the language of salted water and refer to the solid phase of the solvent as ice, to the liquid phase as liquid-water, and to the solute as salt.

\subsection{General Hamiltonian}

Our model will be defined on the $d$-dimensional hypercubic lattice $\mathbb{Z}^{d}$. We will take the (formal) nearest-neighbor Hamiltonian of the following form:

$$
\beta \mathscr{H}=-\sum_{\langle x, y\rangle}\left(\alpha_{\mathrm{I}} I_{x} I_{y}+\alpha_{L} L_{x} L_{y}\right)+\kappa \sum_{x} S_{x} I_{x}-\sum_{x} \mu_{\mathrm{S}} S_{x}-\sum_{x} \mu_{\mathrm{L}} L_{x} .
$$


Here $\beta$ is the inverse temperature (henceforth incorporated into the Hamiltonian), $x$ and $y$ are sites in $\mathbb{Z}^{d}$ and $\langle x, y\rangle$ denotes a neighboring pair of sites. The quantities $I_{x}, L_{x}$ and $S_{x}$ are the ice (water), liquid (water) and salt variables, which will take values in $\{0,1\}$ with the additional constraint

$$
I_{x}+L_{x}=1
$$

valid at each site $x$. We will say that $I_{x}=1$ indicates the presence of ice at $x$ and, similarly, $L_{x}$ the presence of liquid at $x$. Since a single water molecule cannot physically be in an ice state, it is natural to interpret the phrase $I_{x}=1$ as referring to the collective behavior of many particles in the vicinity of $x$ which are enacting an ice-like state, though we do not formally incorporate such a viewpoint into our model.

The various terms in (1.1) are essentially self-explanatory: An interaction between neighboring ice points, similarly for neighboring liquid points (we may assume these to be attractive), an energy penalty $\kappa$ for a simultaneous presence of salt and ice at one point, and, finally, fugacity terms for salt and liquid. For simplicity (and tractability), there is no direct saltsalt interaction, except for the exclusion rule of at most one salt "particle" at each site. Additional terms which could have been included are superfluous due to the constraint (1.2). We will assume throughout that $\kappa>0$, so that the salt-ice interaction expresses the negative affinity of salt to the ice state of water. This term is entirely - and not subtly - responsible for the general phenomenon of freezing point depression. We remark that by suitably renaming the variables, the Hamiltonian in (1.1) would just as well describe a system with boiling point elevation.

As we said, the variables $I_{x}$ and $L_{x}$ indicate the presence of ice and liquid water at site $x$, respectively. The assumption $I_{x}+L_{x}=1$ guarantees that something has to be present at $x$ (the concentration of water in water is unity); what is perhaps unrealistic is the restriction of $I_{x}$ and $L_{x}$ to only the extreme values, namely $I_{x}, L_{x} \in\{0,1\}$. Suffice it to say that the authors are confident (e.g., on the basis of ref. 3) that virtually all the results in this note can be extended to the cases of continuous variables. However, we will not make any such mathematical claims; much of this paper will rely heavily on preexisting technology which, strictly speaking, has only been made to work for the discrete case. A similar discussion applies, of course, to the salt variables. But here our restriction to $S_{x} \in\{0,1\}$ is mostly to ease the exposition; virtually all of our results directly extend to the cases when $S_{x}$ takes arbitrary (positive) real values according to some $a$ priori distribution. 


\subsection{Reduction to Ising Variables}

It is not difficult to see that the "ice-liquid sector" of the general Hamiltonian (1.1) reduces to a ferromagnetic Ising spin system. On a formal level, this is achieved by passing to the Ising variables $\sigma_{x}=L_{x}-I_{x}$, which in light of the constraint (1.2) gives

$$
L_{x}=\frac{1+\sigma_{x}}{2} \quad \text { and } \quad I_{x}=\frac{1-\sigma_{x}}{2} .
$$

By substituting these into (1.1), we arrive at the interaction Hamiltonian:

$$
\beta \mathscr{H}=-J \sum_{\langle x, y\rangle} \sigma_{x} \sigma_{y}-h \sum_{x} \sigma_{x}+\kappa \sum_{x} S_{x} \frac{1-\sigma_{x}}{2}-\sum_{x} \mu_{\mathrm{S}} S_{x},
$$

where the new parameters $J$ and $h$ are given by

$$
J=\frac{\alpha_{\mathrm{L}}+\alpha_{\mathrm{I}}}{4} \quad \text { and } \quad h=\frac{d}{2}\left(\alpha_{\mathrm{L}}-\alpha_{\mathrm{I}}\right)+\frac{\mu_{\mathrm{L}}}{2} .
$$

We remark that the third sum in (1.4) is still written in terms of "ice" indicators so that $\mathscr{H}$ will have a well defined meaning even if $\kappa=\infty$, which corresponds to prohibiting salt entirely at ice-occupied sites. (Notwithstanding, the bulk of this paper is restricted to finite $\kappa$.) Using an appropriate restriction to finite volumes, the above Hamitonian allows us to define the corresponding Gibbs measures. We postpone any relevant technicalities to Section 2.1.

The Hamiltonian as written foretells the possibility of fluctuations in the salt concentration. However, this is not the situation which is of physical interest. Indeed, in an open system it is clear that the salt concentration will, eventually, adjust itself until the system exhibits a pure phase. On the level of the description provided by (1.4) it is noted that, as grand canonical variables, the salt particles can be explicitly integrated, the result being the Ising model at coupling constant $J$ and external field $h_{\text {eff }}$, where

$$
h_{\mathrm{eff}}=h+\frac{1}{2} \log \frac{1+e^{\mu_{\mathrm{S}}}}{1+e^{\mu_{\mathrm{S}}-\kappa}} .
$$

In this context, phase coexistence is confined to the region $h_{\text {eff }}=0$, i.e., a simple curve in the $\left(\mu_{\mathrm{S}}, h\right)$-plane. Unfortunately, as is well known, ${ }^{(5,19,20,23,30)}$ not much insight on the subject of phase separation is to be gained by studying the Ising magnet in an external field. Indeed, under 
(for example) minus boundary conditions, once $h$ exceeds a particular value, a droplet will form which all but subsumes the allowed volume. The transitional value of $h$ scales inversely with the linear size of the system; the exact constants and the subsequent behavior of the droplet depend on the details of the boundary conditions.

The described "failure" of the grand canonical description indicates that the correct ensemble in this case is the one with a fixed amount of salt per unit volume. (The technical definition uses conditioning from the grand canonical measure; see Section 2.1.) This ensemble is physically more relevant because, at the moment of freezing, the salt typically does not have enough "mobility" to be gradually released from the system. It is noted that, once the total amount of salt is fixed, the chemical potential $\mu_{\mathrm{S}}$ drops out of the problem - the relevant parameter is now the salt concentration. As will be seen in Section 2, in our Ising-based model of the solvent-solute system, fixing the salt concentration generically leads to sharp phase separation in the Ising configuration. Moreover, this happens for an interval of values of the magnetic field $h$. Indeed, the interplay between the salt concentration and the actual external field will demand a particular value of the magnetization, even under conditions which will force a droplet (or ice crystal, depending on the boundary condition) into the system.

Remark 1.1. We finish by noting that, while the parameter $h$ is formally unrelated to temperature, it does to a limited extent play the role of temperature in that it reflects the a priori amount of preference of the system for water versus ice. Thus the natural phase diagram to study is in the $(c, h)$-plane.

\subsection{Heuristic Derivations and Outline}

The reasoning which led to formula (1.6) allows for an immediate heuristic explanation of our principal results. The key simplification which again boils down to the absence of salt-salt interaction - is that for any Ising configuration, the amalgamated contribution of salt, i.e., the Gibbs weight summed over salt configurations, depends only on the overall magnetization and not on the details of how the magnetization gets distributed about the system. In systems of linear scale $L$, let $\mathfrak{Z}_{L}(M)$ denote the canonical partition function for the Ising magnet with constrained overall magnetization $M$. The total partition function $Z_{L}(c, h)$ at fixed salt concentration $c$ can then be written as

$$
Z_{L}(c, h)=\sum_{M} \mathfrak{Z}_{L}(M) e^{h M} W_{L}(M, c),
$$


where $W_{L}(M, c)$ denotes the sum of the salt part of the Boltzmann weight - which only depends on the Ising spins via the total magnetization $M-$ over all salt configurations with concentration $c$.

As usual, the physical values of the magnetization are those bringing the dominant contribution to the sum in (1.7). Let us recapitulate the standard arguments by first considering the case $c=0$ (which implies $W_{L}=1$ ), i.e., the usual Ising system at external field $h$. Here we recall that $\mathfrak{Z}_{L}\left(m L^{d}\right)$ can approximately be written as

$$
\mathfrak{Z}_{L}\left(m L^{d}\right) \approx e^{-L^{d}\left[\mathscr{F}_{J}(m)+C\right]},
$$

where $C$ is a suitably chosen constant and $\mathscr{F}_{J}(m)$ is a (normalized) canonical free energy. The principal fact about $\mathscr{F}_{J}(m)$ is that it vanishes for $m$ in the interval $\left[-m_{\star}, m_{\star}\right]$, where $m_{\star}=m_{\star}(J)$ denotes the spontaneous magnetization of the Ising model at coupling $J$, while it is strictly positive and strictly convex for $m$ with $|m|>m_{\star}$. The presence of the "flat piece" on the graph of $\mathscr{F}_{J}(m)$ is directly responsible for the existence of the phase transition in the Ising model: For $h>0$ the dominant contribution to the grand canonical partition function comes from $M \gtrsim m_{\star} L^{d}$ while for $h<0$ the dominant values of the overall magnetization are $M \lesssim-m_{\star} L^{d}$. Thus, once $m_{\star}=m_{\star}(J)>0$ - which happens for $J>J_{\mathrm{c}}(d)$ with $J_{\mathrm{c}}(d) \in(0, \infty)$ whenever $d \geqslant 2-$ a phase transition occurs at $h=0$.

The presence of salt variables drastically changes the entire picture. Indeed, as we will see in Theorem 2.1, the salt partition function $W_{L}(M, c)$ will exhibit a nontrivial exponential behavior which is characterized by a strictly convex free energy. The resulting exponential growth rate of $\mathfrak{Z}_{L}(M) e^{h M} W_{L}(M, c)$ for $M \approx m L^{d}$ is thus no longer a function with a flat piece - instead, for each $h$ there is a unique value of $m$ that optimizes the corresponding free energy. Notwithstanding (again, due to the absence of salt-salt interactions) once that $m$ has been selected, the spin configurations are the typical Ising configurations with overall magnetizations $M \approx$ $m L^{d}$. In particular, whenever $Z_{L}(c, h)$ is dominated by values of $M \approx m L^{d}$ for an $m \in\left(-m_{\star}, m_{\star}\right)$, a macroscopic droplet develops in the system. Thus, due to the one-to-one correspondence between $h$ and the optimal value of $m$, phase separation occurs for an interval of values of $h$ at any positive concentration; see Fig. 1.

We finish with an outline of the remainder of this paper and some discussion of the companion paper. ${ }^{(1)}$ In Section 2, we define precisely the model of interest and state our main results concerning the asymptotic behavior of the corresponding measure on spin and salt configurations with fixed concentration of salt. Along with the results comes 


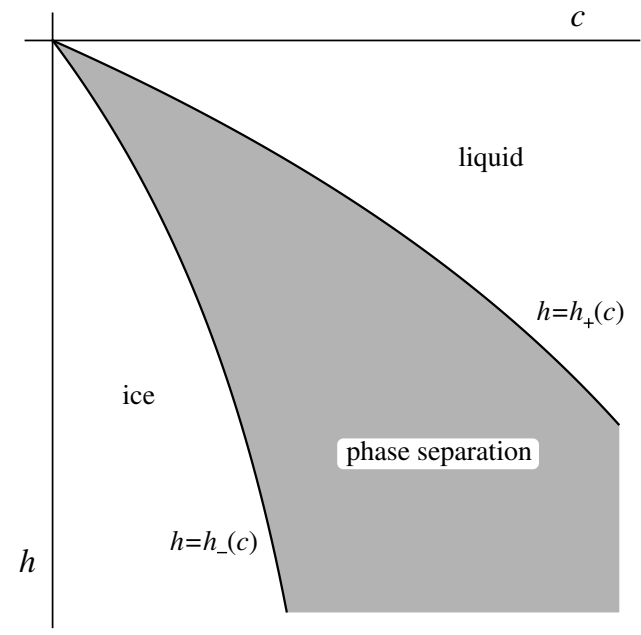

Fig. 1. The phase diagram of the ice-water system with $\kappa \gg 1$. The horizontal axis marks the concentration of the salt in the system, the vertical line represents the external field acting on the Ising spins - see formula (1.5). For positive concentrations $c>0$, the system stays in the liquid-water phase throughout a non-trivial range of negative values of $h$ - a manifestation of the freezing-point depression. For $(h, c)$ in the shaded region, a non-trivial fraction of the system is frozen into ice. Once $(h, c)$ is on the left of the shaded region, the entire system is in the ice state. For moderate values of $\kappa$, the type of convexity of the transition lines may change from concave to convex near $(h, c)=(0,0)$; see the companion paper. ${ }^{(1)}$

a description of the phase diagram and a discussion of freezing-point depression, phase separation, etc., see Section 2.3. Our main results are proved in Section 3. In ref. 1 we investigate the asymptotic of infinitesimal salt concentrations. Interestingly, we find that, in order to induce phase separation, the concentration has to scale at least as the inverse linear size of the system.

\section{RIGOROUS RESULTS}

\subsection{The Model}

With the (formal) Hamiltonian (1.4) in mind, we can now start on developing the mathematical layout of the problem. To define the model, we will need to restrict attention to finite subsets of the lattice. We will mostly focus on rectangular boxes $\Lambda_{L} \subset \mathbb{Z}^{d}$ of $L \times L \times \cdots \times L$ sites centered at the origin. Our convention for the boundary, $\partial \Lambda$, of the set $\Lambda \subset \mathbb{Z}^{d}$ will be the collection of sites outside $\Lambda$ with a neighbor inside $\Lambda$. For each $x \in \Lambda$, we have the water and salt variables, $\sigma_{x} \in\{-1,+1\}$ and $S_{x} \in\{0,1\}$. 
On the boundary, we will consider fixed configurations $\sigma_{\partial \Lambda}$; most of the time we will be discussing the cases $\sigma_{\partial \Lambda}=+1$ or $\sigma_{\partial \Lambda}=-1$, referred to as plus and minus boundary conditions. Since there is no salt-salt interaction, we may as well set $S_{x}=0$ for all $x \in \Lambda^{\mathrm{c}}$.

We will start by defining the interaction Hamiltonian. Let $\Lambda \subset \mathbb{Z}^{d}$ be a finite set. For a spin configuration $\sigma_{\partial \Lambda}$ and the pair $\left(\sigma_{\Lambda}, S_{\Lambda}\right)$ of spin and salt configurations, we let

$$
\beta \mathscr{H}_{\Lambda}\left(\sigma_{\Lambda}, S_{\Lambda} \mid \sigma_{\partial \Lambda}\right)=-J \sum_{\substack{\langle x, y\rangle \\ x \in \Lambda, y \in \mathbb{Z}^{d}}} \sigma_{x} \sigma_{y}-h \sum_{x \in \Lambda} \sigma_{x}+\kappa \sum_{x \in \Lambda} S_{x} \frac{1-\sigma_{x}}{2} .
$$

Here, as before, $\langle x, y\rangle$ denotes a nearest-neighbor pair on $\mathbb{Z}^{d}$ and the parameters $J, h$ and $\kappa$ are as discussed above. (In light of the discussion from Section 1.3 the last term in (1.4) has been omitted.) The probability distribution of the pair $\left(\sigma_{\Lambda}, S_{\Lambda}\right)$ takes the usual Gibbs-Boltzmann form:

$$
P_{\Lambda}^{\sigma_{\partial \Lambda}}\left(\sigma_{\Lambda}, S_{\Lambda}\right)=\frac{e^{-\beta \mathscr{H}_{\Lambda}\left(\sigma_{\Lambda}, S_{\Lambda} \mid \sigma_{\partial \Lambda}\right)}}{Z_{\Lambda}\left(\sigma_{\partial \Lambda}\right)}
$$

where the normalization constant, $Z_{\Lambda}\left(\sigma_{\partial \Lambda}\right)$, is the partition function. The distributions in $\Lambda_{L}$ with the plus and minus boundary conditions will be denoted by $P_{L}^{+}$and $P_{L}^{-}$, respectively.

For reasons discussed before we will be interested in the problems with a fixed salt concentration $c \in[0,1]$. In finite volume, we take this to mean that the total amount of salt,

$$
N_{L}=N_{L}(S)=\sum_{x \in \Lambda_{L}} S_{x}
$$

is fixed. To simplify future discussions, we will adopt the convention that "concentration $c$ " means that $N_{L} \leqslant c\left|\Lambda_{L}\right|<N_{L}+1$, i.e., $N_{L}=\left\lfloor c L^{d}\right\rfloor$. We may then define the finite volume Gibbs probability measure with salt concentration $c$ and plus (or minus) boundary conditions denoted by $P_{L}^{+, c, h}$ (or $P_{L}^{-, c, h}$ ). In light of (2.2), these are given by the formulas

$$
P_{L}^{ \pm, c, h}(\cdot)=P_{L}^{ \pm}\left(\cdot \mid N_{L}=\left\lfloor c L^{d}\right\rfloor\right) .
$$

Both measures $P_{L}^{ \pm, c, h}$ depend on the parameters $J$ and $\kappa$ in the Hamiltonian. However, we will always regard these as fixed and suppress them from the notation whenever possible. 


\subsection{Main Theorems}

In order to describe our first set of results, we will need to bring to bear a few standard facts about the Ising model. For each spin configuration $\sigma=\left(\sigma_{x}\right) \in\{-1,1\}^{\Lambda_{L}}$ let us define the overall magnetization in $\Lambda_{L}$ by the formula

$$
M_{L}=M_{L}(\sigma)=\sum_{x \in \Lambda_{L}} \sigma_{x}
$$

Let $\mathfrak{m}(h, J)$ denote the magnetization of the Ising model with coupling constant $J$ and external field $h \geqslant 0$. As is well known, cf the proof of Theorem 3.1, $h \mapsto \mathfrak{m}(h, J)$ continuously (and strictly) increases from the value of the spontaneous magnetization $m_{\star}=\mathfrak{m}(0, J)$ to one as $h$ sweeps through $[0, \infty)$. In particular, for each $m \in[\mathfrak{m}(0, J), 1)$, there exists a unique $\mathfrak{h}=$ $\mathfrak{h}(m, J) \in[0, \infty)$ such that $\mathfrak{m}(\mathfrak{h}, J)=m$.

Next we will use the above quantities to define the function $\mathscr{F}_{J}:(-1,1) \rightarrow[0, \infty)$, which represents the canonical free energy of the Ising model in (1.8). As it turns out - see Theorem 3.1 in Section 3 we simply have

$$
\mathscr{F}_{J}(m)=\int d m^{\prime} \mathfrak{h}\left(m^{\prime}, J\right) 1_{\left\{m_{\star} \leqslant m^{\prime} \leqslant|m|\right\}}, \quad m \in(-1,1) .
$$

As already mentioned, if $J>J_{\mathrm{c}}$, where $J_{\mathrm{c}}=J_{\mathrm{c}}(d)$ is the critical coupling constant of the Ising model, then $m_{\star}>0$ and thus $\mathscr{F}_{J}(m)=0$ for $m \in$ $\left[-m_{\star}, m_{\star}\right]$. (Since $J_{\mathrm{c}}(d)<\infty$ only for $d \geqslant 2$, the resulting "flat piece" on the graph of $m \mapsto \mathscr{F}_{J}(m)$ appears only in dimensions $d \geqslant 2$.) From the perspective of the large-deviation theory, cf. (refs. 13, 21), $m \mapsto \mathscr{F}_{J}(m)$ is the large-deviation rate function for the magnetization in the (unconstrained) Ising model; see again Theorem 3.1.

Let $\mathscr{S}(p)=p \log p+(1-p) \log (1-p)$ denote the entropy function of the Bernoulli distribution with parameter $p$. (We will set $\mathscr{S}(p)=\infty$ whenever $p \notin[0,1]$.) For each $m \in(-1,1)$, each $c \in[0,1]$ and each $\theta \in[0,1]$, let

$$
\Xi(m, \theta ; c)=-\frac{1+m}{2} \mathscr{S}\left(\frac{2 \theta c}{1+m}\right)-\frac{1-m}{2} \mathscr{S}\left(\frac{2(1-\theta) c}{1-m}\right) .
$$

As we will show in Section 3, this quantity represents the entropy of configurations with fixed salt concentration $c$, fixed overall magnetization $m$ and fixed fraction $\theta$ of the salt residing "on the plus spins" (and fraction $1-\theta$ "on the minus spins"). 
Having defined all relevant quantities, we are ready to state our results. We begin with a large-deviation principle for the magnetization in the measures $P_{L}^{ \pm, c, h}$ :

Theorem 2.1. Let $J>0$ and $\kappa>0$ be fixed. For each $c \in(0,1)$, each $h \in \mathbb{R}$ and each $m \in(-1,1)$, we have

$$
\begin{aligned}
& \lim _{\epsilon \downarrow 0} \lim _{L \rightarrow \infty} \frac{1}{L^{d}} \log P_{L}^{ \pm, c, h}\left(\left|M_{L}-m L^{d}\right| \leqslant \epsilon L^{d}\right) \\
& =-G_{h, c}(m)+\inf _{m^{\prime} \in(-1,1)} G_{h, c}\left(m^{\prime}\right) .
\end{aligned}
$$

Here $m \mapsto G_{h, c}(m)$ is given by

$$
G_{h, c}(m)=\inf _{\theta \in[0,1]} \mathscr{G}_{h, c}(m, \theta),
$$

where

$$
\mathscr{G}_{h, c}(m, \theta)=-h m-\kappa \theta c-\Xi(m, \theta ; c)+\mathscr{F}_{J}(m) .
$$

The function $m \mapsto G_{h, c}(m)$ is finite and strictly convex on $(-1,1)$ with $\lim _{m \rightarrow \pm 1} G_{h, c}^{\prime}(m)= \pm \infty$. Furthermore, the unique minimizer $m=m(h, c)$ of $m \mapsto G_{h, c}(m)$ is continuous in both $c$ and $h$ and strictly increasing in $h$.

On the basis of the above large-deviation result, we can now characterize the typical configurations of the measures $P_{L}^{ \pm, c, h}$. Consider the Ising model with coupling constant $J$ and zero external field and let $\mathbb{P}_{L}^{ \pm, J}$ be the corresponding Gibbs measure in volume $\Lambda_{L}$ and \pm -boundary condition. Our main result in this section is then as follows:

Theorem 2.2. Let $J>0$ and $\kappa>0$ be fixed. Let $c \in(0,1)$ and $h \in$ $\mathbb{R}$, and define two sequences of probability measures $\rho_{L}^{ \pm}$on $[-1,1]$ by the formula

$$
\rho_{L}^{ \pm}([-1, m])=P_{L}^{ \pm, c, h}\left(M_{L} \leqslant m L^{d}\right), \quad m \in[-1,1] .
$$

The measures $\rho_{L}^{ \pm}$allow us to write the spin marginal of the measure $P_{L}^{ \pm, c, h}$ as a convex combination of the Ising measures with fixed magnetization; i.e., for any set $\mathcal{A}$ of configurations $\left(\sigma_{x}\right)_{x \in \Lambda_{L}}$, we have

$$
P_{L}^{ \pm, c, h}\left(\mathcal{A} \times\{0,1\}^{\Lambda_{L}}\right)=\int \rho_{L}^{ \pm}(d m) \mathbb{P}_{L}^{ \pm, J}\left(\mathcal{A} \mid M_{L}=\left\lfloor m L^{d}\right\rfloor\right) .
$$

Moreover, if $m=m(h, c)$ denotes the unique minimizer of the function $m \mapsto G_{h, c}(m)$ from (2.9), then the following properties are true: 
(1) Given the spin configuration on a finite set $\Lambda \subset \mathbb{Z}^{d}$, the salt variables on $\Lambda$ are asymptotically independent. Explicitly, for each finite set $\Lambda \subset \mathbb{Z}^{d}$ and any two configurations $\bar{S}_{\Lambda} \in\{0,1\}^{\Lambda}$ and $\bar{\sigma}_{\Lambda} \in\{-1,1\}^{\Lambda}$,

$$
\begin{aligned}
& \lim _{L \rightarrow \infty} P_{L}^{ \pm, c, h}\left(S_{\Lambda}=\bar{S}_{\Lambda} \mid \sigma_{\Lambda}=\bar{\sigma}_{\Lambda}\right) \\
& \quad=\prod_{x \in \Lambda}\left\{q_{\bar{\sigma}_{x}} \delta_{1}\left(\bar{S}_{x}\right)+\left(1-q_{\bar{\sigma}_{x}}\right) \delta_{0}\left(\bar{S}_{x}\right)\right\},
\end{aligned}
$$

where the numbers $q_{ \pm} \in[0,1]$ are uniquely determined by the equations

$$
\frac{q_{+}}{1-q_{+}}=\frac{q_{-}}{1-q_{-}} e^{\kappa} \quad \text { and } \quad q_{+} \frac{1+m}{2}+q_{-} \frac{1-m}{2}=c .
$$

(2) The measure $\rho_{L}^{ \pm}$converges weakly to a point mass at $m=m(h, c)$,

$$
\lim _{L \rightarrow \infty} \rho_{L}^{ \pm}(\cdot)=\delta_{m}(\cdot)
$$

In particular, the Ising-spin marginal of the measure $P_{L}^{ \pm, c, h}$ is asymptotically supported on the usual Ising spin configurations with the overall magnetization $M_{L}=(m+o(1)) L^{d}$, where $m$ minimizes $m \mapsto G_{h, c}(m)$.

The fact that conditioning $P_{L}^{ \pm, c, h}$ on a fixed value of magnetization produces the Ising measure under same conditioning - which is the content of (2.12) - is directly related to the absence of salt-salt interaction. The principal conclusions of the previous theorem are thus parts (1) and (2), which state that the presence of a particular amount of salt forces the Ising sector to choose a particular value of magnetization density. The underlying variational principle provides insight into the physical mechanism of phase separation upon freezing of solutions. (We refer the reader back to Section 1.4 for the physical basis of these considerations.)

We will proceed by discussing the consequences of these results for the phase diagram of the model and, in particular, the phenomenon of freezing point depression. Theorems 2.1 and 2.2 are proved in Section 3.2.

\subsection{Phase Diagram}

The representation (2.12) along with the asymptotic (2.15) allow us to characterize the distribution $P_{L}^{ \pm, c, h}$ in terms of the canonical ensemble of the Ising ferromagnet. Indeed, these formulas imply that the distribution of Ising spins induced by $P_{L}^{ \pm, c, h}$ is very much like that in the 
measure $\mathbb{P}_{L}^{ \pm, J}$ conditioned on the event that the overall magnetization $M_{L}$ is near the value $m(h, c) L^{d}$. As a consequence, the asymptotic statements (e.g., the Wulff construction) that have been (or will be) established for the spin configurations in the Ising model with fixed magnetization will automatically hold for the spin-marginal of the $P_{L}^{ \pm, c, h}$ as well.

A particular question of interest in this paper is phase separation. Recall that $m_{\star}=m_{\star}(J)$ denotes the spontaneous magnetization of the Ising model at coupling $J$. Then we can anticipate the following conclusions about typical configurations in measure $P_{L}^{ \pm, c, h}$ :

(1) If $m(h, c) \geqslant m_{\star}$, then the entire system (with plus boundary condition) will look like the plus state of the Ising model whose external field is adjusted so that the overall magnetization on the scale $L^{d}$ is roughly $m(h, c) L^{d}$.

(2) If $m(h, c) \leqslant-m_{\star}$, then the system (with minus boundary condition) will look like the Ising minus state with similarly adjusted external field.

(3) If $m(h, c) \in\left(-m_{\star}, m_{\star}\right)$, then, necessarily, the system exhibits phase separation in the sense that typical configurations feature a large droplet of one phase inside the other. The volume fraction taken by the droplet is such that the overall magnetization is near $m(h, c) L^{d}$. The outer phase of the droplet agrees with the boundary condition.

The cases (1-2) with opposite boundary conditions - that is, the minus boundary conditions in (1) and the plus boundary conditions in (2) - are still as stated; the difference is that now there has to be a large contour near the boundary flipping to the "correct" boundary condition.

Remark 2.3. We have no doubt that the aforementioned conclusions (1-3) hold for all $d \geqslant 2$ and all $J>J_{\mathrm{c}}$ (with a proper definition of the droplet in part (3), of course). However, the depth of conclusion (3) depends on the level of understanding Wulff construction, which is at present rather different in dimensions $d=2$ and $d \geqslant 3$. Specifically, while in $d=2$ the results of ${ }^{(14,22)}$ allow us to claim that for all $J>J_{\mathrm{c}}$ and all magnetizations $m \in\left(-m_{\star}, m_{\star}\right)$, the system will exhibit a unique large contour with appropriate properties, in $d \geqslant 3$ this statement is known to hold $\mathrm{d}^{(6,10)}$ only in " $L$-sense" and only for $m \in\left(-m_{\star}, m_{\star}\right)$ which are near the endpoints. (Moreover, not all values of $J>J_{\mathrm{c}}$ are, in principle, permitted; cf. ref. 7 for a recent improvement of these restrictions.) We refer to ref. 8 for an overview of the situation.

Notwithstanding the technical difficulties of Wulff construction, the above allows us to characterize the phase diagram of the model at hand. 
As indicated in Fig. 1 , the $h \leqslant 0$ and $c \geqslant 0$ quadrant splits into three distinct parts: The liquid-water region, the ice region and the phase separation region, which correspond to the situations in (1-3), respectively. The boundary lines of the phase-separation region are found by setting

$$
m(h, c)= \pm m_{\star},
$$

which in light of strict monotonicity of $h \mapsto m(h, c)$ allows us to calculate $h$ as a function of $c$. The solutions of (2.16) can be obtained on the basis of the following observation:

Proposition 2.4. Let $m \in\left[-m_{\star}, m_{\star}\right]$ and $c \in[0,1]$ and define the quantities $q_{ \pm}=q_{ \pm}(m, c, \kappa)$ by (2.14). Let $h$ be the solution to $m(h, c)=m$. Then we have:

$$
h=\frac{1}{2} \log \frac{1-q_{+}}{1-q_{-}} .
$$

In particular, there exist two continuous and decreasing functions $h_{ \pm}$: $[0, \infty) \rightarrow(-\infty, 0]$ with $h_{+}(c)>h_{-}(c)$ for all $c>0$, such that $-m_{\star}<$ $m(h, c)<m_{\star}$ is equivalent to $h_{-}(c)<h<h_{+}(c)$ for all $c>0$.

Proposition 2.4 is proved at the very end of Section 3.2. Here is an informal interpretation of this result: The quantities $q_{ \pm}$represent the mole fractions of salt in liquid-water and ice, respectively. In mathematical terms, $q_{+}$is the probability of having a salt particle on a given plus spin, and $q_{-}$is the corresponding quantity for minus spins, see (2.13). Formula (2.17) quantifies the shift of the chemical potential of the solvent (which is given by $2 h$ in this case) due to the presence of the solute. This is a manifestation of freezing-point depression, see also Remark 1.1. In the asymptotic when $c \ll 1$ we have

$$
2 h \approx q_{-}-q_{+} .
$$

This relation, derived in standard chemistry and physics books under the auspicies of the "usual approximations," is an essential ingredient in the classical analyses of colligative properties of solutions. ${ }^{(24,27)}$ Here the derivation is a direct consequence of a microscopic (albeit simplistic) model which further offers the possibility of systematically calculating higherorder corrections. 


\section{PROOFS}

The proofs of our main results are, more or less, straightforward exercises in large-deviation analysis of Gibbs distributions. We first state and prove a couple of technical lemmas; the actual proofs come in Section 3.2.

\subsection{Preliminaries}

The starting point of the proof of Theorem 2.1 (and, consequently, Theorem 2.2) is the following large-deviation principle for the Ising model at zero external field:

Theorem 3.1. Consider the Ising model with coupling constant $J \in$ $[0, \infty)$ and zero external field. Let $\mathbb{P}_{L}^{ \pm, J}$ be the corresponding (grand canonical) Gibbs measure in volume $\Lambda_{L}$ and \pm -boundary conditions. Then for all $m \in[-1,1]$,

$$
\lim _{\epsilon \downarrow 0} \lim _{L \rightarrow \infty} \frac{1}{L^{d}} \log \mathbb{P}_{L}^{ \pm, J}\left(\left|M_{L}-m L^{d}\right| \leqslant \epsilon L^{d}\right)=-\mathscr{F}_{J}(m),
$$

where $M_{L}$ is as in (2.5) and $\mathscr{F}_{J}$ is as defined in (2.6).

Proof. The claim is considered standard, see e.g. (ref. 31, Section II.1), and follows by a straightforward application of the thermodynamic relations between the free energy, magnetization and external field. For completeness (and reader's convenience) we will provide a proof.

Consider the function $\phi_{L}(h)=\frac{1}{L^{d}} \log \mathbb{E}_{L}^{ \pm, J}\left(e^{h M_{L}}\right)$, where $\mathbb{E}_{L}^{ \pm, J}$ is the expectation with respect to $\mathbb{P}_{L}^{ \pm, J}$, and let $\phi(h)=\lim _{L \rightarrow \infty} \phi_{L}(h)$. The limit exists by subadditivity arguments and is independent of the boundary condition. The function $h \mapsto \phi(h)$ is convex on $\mathbb{R}$, real analytic (by the LeeYang theorem ${ }^{(25)}$ ) on $\mathbb{R} \backslash\{0\}$, and hence it is strictly convex on $\mathbb{R}$. By the $h \leftrightarrow-h$ symmetry there is a cusp at $h=0$ whenever $m_{\star}=\phi^{\prime}\left(0^{+}\right)>0$. It follows that for each $m \in\left[m_{\star}, 1\right)$ there is a unique $\mathfrak{h}=\mathfrak{h}(m, J) \geqslant 0$ such that $\phi^{\prime}(\mathfrak{h})=m$, with $\mathfrak{h}(m, J)$ increasing continuously from 0 to $\infty$ as $m$ increases from $m_{\star}$ to 1 . The plus-minus symmetry shows that a similar statement holds for the magnetizations in $\left(-1,-m_{\star}\right]$.

Let $\phi^{\star}$ denote the Legendre transform of $\phi$, i.e., $\phi^{\star}(m)=\sup _{h \in \mathbb{R}}[m h-$ $\phi(h)$ ]. By the above properties of $h \mapsto \phi(h)$ we infer that $\phi^{\star}(m)=m \mathfrak{h}-\phi(\mathfrak{h})$ when $m \in\left(-1,-m_{\star}\right) \cup\left(m_{\star}, 1\right)$ and $\mathfrak{h}=\mathfrak{h}(m, J)$, while $\phi^{\star}(m)=-\phi(0)=0$ for $m \in\left[-m_{\star}, m_{\star}\right]$. Applying the Gärtner-Ellis theorem (ref. 21, Theorem V.6 or ref. 13, Theorem 2.3.6), we then have (3.1) with $\mathscr{F}_{J}(m)=\phi^{\star}(m)$ for all $m \in\left[-1,-m_{\star}\right) \cup\left(m_{\star}, 1\right]-$ which is the set of so called exposed points of $\phi^{\star}$. Since $\phi^{\star}\left( \pm m_{\star}\right)=0$ and the derivative of $m \mapsto \phi^{\star}(m)$ is $\mathfrak{h}(m, J)$, this $\mathscr{F}_{J}$ 
is given by the integral in (2.6). To prove (3.1) when $m \in\left[-m_{\star}, m_{\star}\right]$, we must note that the left-hand-side of (3.1) is nonpositive and concave in $m$. (This follows by partitioning $\Lambda_{L}$ into two parts with their own private magnetizations and disregarding the interaction through the boundary.) Since $\mathscr{F}_{J}(m)$ tends to zero as $m$ tends to $\pm m_{\star}$ we thus have that (3.1) for $m \in\left[-m_{\star}, m_{\star}\right]$ as well.

Remark 3.2. The "first" part of the Gärtner-Ellis theorem (ref. 21, Theorem V.6) actually guarantees the following large-deviation principle:

$$
\limsup _{L \rightarrow \infty} \frac{1}{L^{d}} \log \mathbb{P}_{L}^{ \pm, J}\left(\frac{M_{L}}{L^{d}} \in \mathcal{C}\right) \leqslant-\inf _{m \in \mathcal{C}} \phi^{\star}(m)
$$

for any closed set $\mathcal{C} \subset \mathbb{R}$ while

$$
\liminf _{L \rightarrow \infty} \frac{1}{L^{d}} \log \mathbb{P}_{L}^{ \pm, J}\left(\frac{M_{L}}{L^{d}} \in \mathcal{O}\right) \geqslant-\inf _{m \in \mathcal{O} \backslash\left[-m_{\star}, m_{\star}\right]} \phi^{\star}(m)
$$

for any open set $\mathcal{O} \subset \mathbb{R}$. (Here $\phi^{\star}(m)=\mathscr{F}_{J}(m)$ for $m \in[-1,1]$ and $\phi^{\star}(m)=$ $\infty$ otherwise.) The above proof follows by specializing to $\epsilon$-neighborhoods of a given $m$ and letting $\epsilon \downarrow 0$. The $m \in\left[-m_{\star}, m_{\star}\right]$ cases - i.e, the nonexposed points - have to be dealt with separately.

The above is the core of our proof of Theorem 2.1. The next step will be to bring the quantities $c$ and $h$ into play. This, as we shall see, is easily done if we condition on the total magnetization. (The cost of this conditioning will be estimated by (3.1).) Indeed, as a result of the absence of salt-salt interaction, the conditional measure can be rather precisely characterized. Let us recall the definition of the quantity $N_{L}$ from (2.3) which represents the total amount of salt in the system. For any spin configuration $\sigma=\left(\sigma_{x}\right) \in\{-1,1\}^{\Lambda_{L}}$ and any salt configuration $S=\left(S_{x}\right) \in\{0,1\}^{\Lambda_{L}}$, let us introduce the quantity

$$
Q_{L}=Q_{L}(\sigma, S)=\sum_{x \in \Lambda_{L}} S_{x} \frac{1+\sigma_{x}}{2}
$$

representing the total amount of salt "on the plus spins." Then we have:

Lemma 3.3. For any fixed spin configuration $\bar{\sigma}=\left(\bar{\sigma}_{x}\right) \in\{-1,1\}^{\Lambda_{L}}$, all salt configurations $\left(S_{x}\right) \in\{0,1\}^{\Lambda_{L}}$ with the same $N_{L}$ and $Q_{L}$ have the 
same probability in the conditional measure $P_{L}^{ \pm, c, h}(\cdot \mid \sigma=\bar{\sigma})$. Moreover, for any $\bar{S}=\left(\bar{S}_{x}\right) \in\{0,1\}^{\Lambda_{L}}$ with $N_{L}=\left\lfloor c L^{d}\right\rfloor$ and for any $m \in[-1,1]$,

$$
\begin{aligned}
& P_{L}^{ \pm, c, h}\left(\bar{S} \text { occurs, } M_{L}=\left\lfloor m L^{d}\right\rfloor\right) \\
& \quad=\frac{1}{Z_{L}} \mathbb{E}_{L}^{ \pm, J}\left(e^{\kappa Q_{L}(\sigma, \bar{S})+h M_{L}(\sigma)} 1_{\left\{M_{L}(\sigma)=\left\lfloor m L^{d}\right\rfloor\right\}}\right),
\end{aligned}
$$

where the normalization constant is given by

$$
Z_{L}=\sum_{S^{\prime} \in\{0,1\}^{\Lambda} L} 1_{\left\{N_{L}\left(S^{\prime}\right)=\left\lfloor c L^{d}\right\rfloor\right\}} \mathbb{E}_{L}^{ \pm, J}\left(e^{\kappa Q_{L}\left(\sigma, S^{\prime}\right)+h M_{L}(\sigma)}\right)
$$

Here $\mathbb{E}_{L}^{ \pm, J}$ is the expectation with respect to $\mathbb{P}_{L}^{ \pm, J}$.

Proof. The fact that all salt configurations with given $N_{L}$ and $Q_{L}$ have the same probability in $P_{L}^{ \pm, c, h}(\cdot \mid \sigma=\bar{\sigma})$ is a consequence of the observation that the salt-dependent part of the Hamiltonian (2.1) depends only on $Q_{L}$. The relations (3.5)-(3.6) follow by a straightforward rewrite of the overall Boltzmann weight.

The characterization of the conditional measure $P_{L}^{ \pm, c, h}\left(\cdot \mid M_{L}=\left\lfloor m L^{d}\right\rfloor\right)$ from Lemma 3.3 allows us to explicitly evaluate the configurational entropy carried by the salt. Specifically, given a spin configuration $\sigma=$ $\left(\sigma_{x}\right) \in\{-1,1\}^{\Lambda_{L}}$ and numbers $\theta, c \in(0,1)$, let

$$
\mathcal{A}_{L}^{\theta, c}(\sigma)=\left\{\left(S_{x}\right) \in\{0,1\}^{\Lambda_{L}}: N_{L}=\left\lfloor c L^{d}\right\rfloor, Q_{L}=\left\lfloor\theta c L^{d}\right\rfloor\right\} .
$$

The salt entropy is then the rate of exponential growth of the size of $\mathcal{A}_{L}^{\theta, c}(\sigma)$ which can be related to the quantity $\Xi(m, \theta ; c)$ from $(2.7)$ as follows:

Lemma 3.4. For each $\epsilon^{\prime}>0$ and each $\eta>0$ there exists a number $L_{0}<\infty$ such that the following is true for any $\theta, c \in(0,1)$, any $m \in$ $(-1,1)$ that obey $|m| \leqslant 1-\eta$,

$$
\frac{2 \theta c}{1+m} \leqslant 1-\eta \quad \text { and } \quad \frac{2(1-\theta) c}{1-m} \leqslant 1-\eta
$$

and any $L \geqslant L_{0}$ : If $\sigma=\left(\sigma_{x}\right) \in\{-1,1\}^{\Lambda_{L}}$ is a spin configuration with $M_{L}(\sigma)=\left\lfloor m L^{d}\right\rfloor$, then

$$
\left|\frac{\log \left|\mathcal{A}_{L}^{\theta, c}(\sigma)\right|}{L^{d}}-\Xi(m, \theta ; c)\right| \leqslant \epsilon^{\prime}
$$


Proof. We want to distribute $N_{L}=\left\lfloor c L^{d}\right\rfloor$ salt particles over $L^{d}$ positions, such that exactly $Q_{L}=\left\lfloor\theta c L^{d}\right\rfloor$ of them land on $\frac{1}{2}\left(L^{d}+M_{L}\right)$ plus sites and $N_{L}-Q_{L}$ on $\frac{1}{2}\left(L^{d}-M_{L}\right)$ minus sites. This can be done in

$$
\left|\mathcal{A}_{L}^{\theta, c}(\sigma)\right|=\left(\begin{array}{c}
\frac{1}{2}\left(L^{d}+M_{L}\right) \\
Q_{L}
\end{array}\right)\left(\begin{array}{c}
\frac{1}{2}\left(L^{d}-M_{L}\right) \\
N_{L}-Q_{L}
\end{array}\right)
$$

number of ways. Now all quantities scale proportionally to $L^{d}$ which, applying Stirling's formula, shows that the first term is within, say, $e^{ \pm L^{d} \epsilon^{\prime} / 2}$ multiples of

$$
\exp \left\{-L^{d} \frac{1+m}{2} \mathscr{S}\left(\frac{2 \theta c}{1+m}\right)\right\}
$$

once $L \geqslant L_{0}$, with $L_{0}$ depending only on $\epsilon^{\prime}$. A similar argument holds also for the second term with $\theta$ replaced by $1-\theta$ and $m$ by $-m$. Combining these expressions we get that $\left|\mathcal{A}_{L}^{\theta, c}(\sigma)\right|$ is within $e^{ \pm L^{d} \epsilon^{\prime}}$ multiples of $\exp \left\{L^{d} \Xi(m, \theta ; c)\right\}$ once $L$ is sufficiently large.

For the proof of Theorem 2.2, we will also need an estimate on how many salt configurations in $\mathcal{A}_{L}^{\theta, c}(\sigma)$ take given values in a finite subset $\Lambda \subset$ $\Lambda_{L}$. To that extent, for each $\sigma \in\{-1,1\}^{\Lambda_{L}}$ and each $\bar{S}_{\Lambda} \in\{0,1\}^{\Lambda}$ we will define the quantity

$$
R_{\Lambda, L}^{\theta, c}\left(\sigma, \bar{S}_{\Lambda}\right)=\frac{\left|\left\{\bar{S} \in \mathcal{A}_{L}^{\theta, c}(\sigma): S_{\Lambda}=\bar{S}_{\Lambda}\right\}\right|}{\left|\mathcal{A}_{L}^{\theta, c}(\sigma)\right|} .
$$

As a moment's thought reveals, $R_{\Lambda, L}^{\theta, c}\left(\sigma, \bar{S}_{\Lambda}\right)$ can be interpreted as the probability that $\left\{S_{\Lambda}=\bar{S}_{\Lambda}\right\}$ occurs in (essentially) any homogeneous product measure on $S=\left(S_{x}\right) \in\{0,1\}^{\Lambda_{L}}$ conditioned to have $N_{L}(S)=\left\lfloor c L^{d}\right\rfloor$ and $Q_{L}(\sigma, S)=\left\lfloor\theta c L^{d}\right\rfloor$. It is therefore not surprising that, for spin configurations $\sigma$ with given magnetization, $R_{\Lambda, L}^{\theta, c}(\sigma, \cdot)$ will tend to a product measure on $S_{\Lambda} \in\{0,1\}^{\Lambda}$. A precise characterization of this limit is as follows:

Lemma 3.5. For each $\epsilon>0$, each $K \geqslant 1$ and each $\eta>0$ there exists $L_{0}<\infty$ such that the following holds for all $L \geqslant L_{0}$, all $\Lambda \subset \Lambda_{L}$ with $|\Lambda| \leqslant K$, all $m$ with $|m| \leqslant 1-\eta$ and all $\theta, c \in[\eta, 1-\eta]$ for which

$$
p_{+}=\frac{2 \theta c}{1+m} \quad \text { and } \quad p_{-}=\frac{2(1-\theta) c}{1-m}
$$


satisfy $p_{ \pm} \in[\eta, 1-\eta]$ : If $\sigma=\left(\sigma_{x}\right) \in\{-1,1\}^{\Lambda_{L}}$ is a spin configuration such that $M_{L}(\sigma)=\left\lfloor m L^{d}\right\rfloor$ and $\bar{S}_{\Lambda} \in\{0,1\}^{\Lambda}$ is a salt configuration in $\Lambda$, then

$$
\left|R_{\Lambda, L}^{\theta, c}\left(\sigma, S_{\Lambda}\right)-\prod_{x \in \Lambda}\left\{p_{\sigma_{x}} \delta_{1}\left(\bar{S}_{x}\right)+\left(1-p_{\sigma_{x}}\right) \delta_{0}\left(\bar{S}_{x}\right)\right\}\right| \leqslant \epsilon .
$$

Proof. We will expand on the argument from Lemma 3.4. Indeed, from (3.10) we have an expression for the denominator in (3.12). As to the numerator, introducing the quantities

$$
M_{\Lambda}=\sum_{x \in \Lambda} \sigma_{x}, \quad N_{\Lambda}=\sum_{x \in \Lambda} S_{x}, \quad Q_{\Lambda}=\sum_{x \in \Lambda} S_{x} \frac{1+\sigma_{x}}{2}
$$

and the shorthand

$$
D=D_{r, r^{\prime}, s, s^{\prime}}\left(\ell, \ell^{\prime}, q, q^{\prime}\right)=\frac{\left(\begin{array}{c}
r-\ell \\
s-q
\end{array}\right)\left(\begin{array}{l}
r^{\prime}-\ell^{\prime} \\
s^{\prime}-q^{\prime}
\end{array}\right)}{\left(\begin{array}{l}
r \\
s
\end{array}\right)\left(\begin{array}{l}
r^{\prime} \\
s^{\prime}
\end{array}\right)}
$$

the same reasoning as we used to prove (3.10) allows us to write the object $R_{\Lambda, L}^{\theta, c}\left(\sigma, S_{\Lambda}\right)$ as $D_{r, r^{\prime}, s, s^{\prime}}\left(\ell, \ell^{\prime}, q, q^{\prime}\right)$, where the various parameters are as follows: The quantities

$$
r=\frac{L^{d}+M_{L}}{2} \quad \text { and } \quad r^{\prime}=\frac{L^{d}-M_{L}}{2}
$$

represent the total number of pluses and minuses in the system, respectively,

$$
s=Q_{L} \quad \text { and } \quad s^{\prime}=N_{L}-Q_{L}
$$

are the numbers of salt particles on pluses and minuses, and, finally,

$$
\ell=\frac{|\Lambda|+M_{\Lambda}}{2}, \quad \ell^{\prime}=\frac{|\Lambda|-M_{\Lambda}}{2}, \quad q=Q_{\Lambda} \quad \text { and } \quad q^{\prime}=N_{\Lambda}-Q_{\Lambda}
$$

are the corresponding quantities for the volume $\Lambda$, respectively.

Since (3.13) and the restrictions on $|m| \leqslant 1-\eta$ and $\theta, c \in[\eta, 1-\eta]$ imply that $r, r^{\prime}, s, s^{\prime}, r-s$ and $r^{\prime}-s^{\prime}$ all scale proportionally to $L^{d}$, uniformly in $\sigma$ and $S_{\Lambda}$, while $\ell$ and $\ell^{\prime}$ are bounded by $|\Lambda|-$ which by our 
assumption is less than $K$ - we are in a regime where it makes sense to seek an asymptotic form of quantity $D$. Using the bounds

$$
a^{b} e^{-b^{2} / a} \leqslant \frac{(a+b) !}{a !} \leqslant a^{b} e^{b^{2} / a},
$$

which are valid for all integers $a$ and $b$ with $|b| \leqslant a$, we easily find that

$$
D=\left(\frac{s}{r}\right)^{\ell}\left(1-\frac{s}{r}\right)^{\ell-q}\left(\frac{s^{\prime}}{r^{\prime}}\right)^{\ell^{\prime}}\left(1-\frac{s^{\prime}}{r^{\prime}}\right)^{\ell^{\prime}-q^{\prime}}+o(1), \quad L \rightarrow \infty .
$$

Since $s / r \rightarrow p_{+}$and $s^{\prime} / r^{\prime} \rightarrow p_{-}$as $L \rightarrow \infty$, while $\ell, q, \ell^{\prime}$ and $q^{\prime}$ stay bounded, the desired claim follows by taking $L$ sufficiently large.

The reader may have noticed that, in most of our previous arguments, $\theta$ and $m$ were restricted to be away from the boundary values. To control the situation near the boundary values, we have to prove the following claim:

Lemma 3.6. For each $\epsilon \in(0,1)$ and each $L \geqslant 1$, let $\mathcal{E}_{L, \epsilon}$ be the event

$$
\begin{aligned}
\mathcal{E}_{L, \epsilon} & =\left\{\left|M_{L}\right| \leqslant(1-\epsilon) L^{d}\right\} \\
& \cap\left\{\epsilon \frac{1}{2}\left(L^{d}+M_{L}\right) \leqslant Q_{L} \leqslant(1-\epsilon) \frac{1}{2}\left(L^{d}+M_{L}\right)\right\} .
\end{aligned}
$$

Then for each $c \in(0,1)$ and each $h \in \mathbb{R}$ there exists an $\epsilon>0$ such that

$$
\limsup _{L \rightarrow \infty} \frac{1}{L^{d}} \log P_{L}^{ \pm, c, h}\left(\mathcal{E}_{L, \epsilon}^{\mathrm{c}}\right)<0 .
$$

Proof. We will split the complement of $\mathcal{E}_{L, \epsilon}$ into four events and prove the corresponding estimate for each of them. We begin with the event $\left\{M_{L} \leqslant-(1-\epsilon) L^{d}\right\}$. The main tool will be stochastic domination by a product measure. Consider the usual partial order on spin configurations defined by putting $\sigma \prec \sigma^{\prime}$ whenever $\sigma_{x} \leqslant \sigma_{x}^{\prime}$ for all $x$. Let

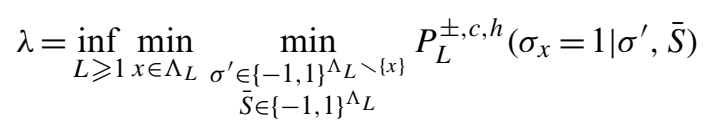

be the conditional probability that $\sigma_{x}=+1$ occurs given a spin configuration $\sigma^{\prime}$ in $\Lambda_{L} \backslash\{x\}$ and a salt configuration $\bar{S}$ in $\Lambda_{L}$, optimized over all $\sigma^{\prime}$, $\bar{S}$ and also $x \in \Lambda_{L}$ and the system size. Since $P_{L}^{ \pm, c, h}\left(\sigma_{x}=1 \mid \sigma^{\prime}, \bar{S}\right)$ reduces 
to (the exponential of) the local interaction between $\sigma_{x}$ and its ultimate neighborhood, we have $\lambda>0$.

Using standard arguments it now follows that the spin marginal of $P_{L}^{ \pm, c, h}$ stochastically dominates the product measure $\mathbb{P}_{\lambda}$ defined by $\mathbb{P}_{\lambda}\left(\sigma_{x}=1\right)=\lambda$ for all $x$. In particular, we have

$$
P_{L}^{ \pm, c, h}\left(M_{L} \leqslant-(1-\epsilon) L^{d}\right) \leqslant \mathbb{P}_{\lambda}\left(M_{L} \leqslant-(1-\epsilon) L^{d}\right)
$$

Let $\epsilon<2 \lambda$. Then $\lambda-(1-\lambda)-$ namely, the expectation of $\sigma_{x}$ with respect to $\mathbb{P}_{\lambda}$ - exceeds the negative of $(1-\epsilon)$ and so Cramér's theorem (ref. 21, Theorem I.4 or ref. 13, Theorem 2.1.24) implies that the probability on the right-hand-side decays to zero exponentially in $L^{d}$, i.e.,

$$
\limsup _{L \rightarrow \infty} \frac{1}{L^{d}} \log \mathbb{P}_{\lambda}\left(M_{L} \leqslant-(1-\epsilon) L^{d}\right)<0 .
$$

The opposite side of the interval of magnetizations, namely, the event $\left\{M_{L} \geqslant(1-\epsilon) L^{d}\right\}$, is handled analogously (with $\lambda$ now focusing on $\sigma_{x}=-1$ instead of $\left.\sigma_{x}=1\right)$.

The remaining two events, marking when $Q_{L}$ is either less than $\epsilon$ or larger than $(1-\epsilon)$ times the total number of plus spins, are handled using a similar argument combined with standard convexity estimates. Let us consider the event $\left\{Q_{L} \leqslant \epsilon L^{d}\right\}-$ which contains the event $\left\{Q_{L} \leqslant \epsilon \frac{1}{2}\left(M_{L}+\right.\right.$ $\left.\left.L^{d}\right)\right\}$ - and let us emphasize the dependence of the underlying probability distribution on $\kappa$ by writing $P_{L}^{ \pm, c, h}$ as $\mathbb{P}_{\kappa}$. Let $\mathbb{E}_{\kappa}$ denote the expectation with respect to $\mathbb{P}_{\kappa}$ and note that $\mathbb{E}_{\kappa}(f)=\mathbb{E}_{0}\left(f e^{\kappa} Q_{L}\right) / \mathbb{E}_{0}\left(e^{\kappa} Q_{L}\right)$. We begin by using the Chernoff bound to get

$$
\mathbb{P}_{\kappa}\left(Q_{L} \leqslant \epsilon L^{d}\right) \leqslant e^{a \epsilon L^{d}} \mathbb{E}_{\kappa}\left(e^{-a Q_{L}}\right)=\frac{e^{a \epsilon L^{d}}}{\mathbb{E}_{\kappa-a}\left(e^{a Q_{L}}\right)}, \quad a \geqslant 0
$$

A routine application of Jensen's inequality gives us

$$
\mathbb{P}_{\kappa}\left(Q_{L} \leqslant \epsilon L^{d}\right) \leqslant \exp \left\{a\left(\epsilon L^{d}-\mathbb{E}_{\kappa-a}\left(Q_{L}\right)\right)\right\}
$$

It thus suffices to prove that there exists a $\kappa^{\prime}<\kappa$ such that inf $L_{L} \geqslant 1 \frac{1}{L^{d}} \mathbb{E}_{\kappa^{\prime}}\left(Q_{L}\right)$ is positive. (Indeed, we take $\epsilon$ to be strictly less than this number and set $a=\kappa-\kappa^{\prime}$ to observe that the right-hand-side decays exponentially in $L^{d}$.) To show this we write $\mathbb{E}_{\kappa^{\prime}}\left(Q_{L}\right)$ as the sum of $\mathbb{P}_{\kappa^{\prime}}\left(\sigma_{x}=1, S_{x}=1\right)$ over all $x \in \Lambda_{L}$. 
Looking back at (3.24), we then have $\mathbb{P}_{\kappa^{\prime}}\left(\sigma_{x}=1, S_{x}=1\right) \geqslant \lambda \mathbb{P}_{\kappa^{\prime}}\left(S_{x}=1\right)$, where $\lambda$ is now evaluated for $\kappa^{\prime}$, and so

$$
\mathbb{E}_{\kappa^{\prime}}\left(Q_{L}\right) \geqslant \lambda \sum_{x \in \Lambda_{L}} \mathbb{P}_{\kappa^{\prime}}\left(S_{x}=1\right)=\lambda \mathbb{E}_{\kappa^{\prime}}\left(N_{L}\right) \approx \lambda c L^{d}
$$

Thus, once $\lambda c>\epsilon$, the probability $\mathbb{P}_{\kappa}\left(Q_{L} \leqslant \epsilon L^{d}\right)$ decays exponentially in $L^{d}$.

As to the complementary event, $\left\{Q_{L} \geqslant(1-\epsilon) \frac{1}{2}\left(M_{L}+L^{d}\right)\right\}$, we note that this is contained in $\left\{H_{L} \leqslant \epsilon L^{d}\right\}$, where $H_{L}$ counts the number of plus spins with no salt on it. Since we still have $\mathbb{E}_{\kappa}(f)=$ $\mathbb{E}_{0}\left(f e^{-\kappa H_{L}}\right) / \mathbb{E}_{0}\left(e^{-\kappa H_{L}}\right)$, the proof boils down to the same argument as before.

\subsection{Proofs of Theorems 2.1 and 2.2}

On the basis of the above observations, the proofs of our main theorems are easily concluded. However, instead of Theorem 2.1 we will prove a slightly stronger result of which the large-deviation part of Theorem 2.1 is an easy corollary.

Theorem 3.7. Let $J>0$ and $\kappa \geqslant 0$ be fixed. For each $c, \theta \in(0,1)$, each $h \in \mathbb{R}$ and each $m \in(-1,1)$, let $\mathcal{B}_{L, \epsilon}=\mathcal{B}_{L, \epsilon}(m, c, \theta)$ be the set of all $(\sigma, S) \in\{-1,1\}^{\Lambda_{L}} \times\{0,1\}^{\Lambda_{L}}$ for which $\left|M_{L}-m L^{d}\right| \leqslant \epsilon L^{d}$ and $\left|Q_{L}-\theta c L^{d}\right| \leqslant$ $\epsilon L^{d}$ hold. Then

$$
\lim _{\epsilon \downarrow 0} \lim _{L \rightarrow \infty} \frac{\log P_{L}^{ \pm, c, h}\left(\mathcal{B}_{L, \epsilon}\right)}{L^{d}}=-\mathscr{G}_{h, c}(m, \theta)+\inf _{\substack{m^{\prime} \in(-1,1) \\ \theta^{\prime} \in[0,1]}} \mathscr{G}_{h, c}\left(m^{\prime}, \theta^{\prime}\right),
$$

where $\mathscr{G}_{h, c}(m, \theta)$ is as in (2.10).

Proof. Since the size of the set $\mathcal{A}_{L}^{\theta, c}(\sigma)$ depends only on the overall magnetization, let $A_{L}^{\theta, c}(m)$ denote this size for the configurations $\sigma$ with $M_{L}(\sigma)=\left\lfloor m L^{d}\right\rfloor$. First we note that, by Lemma 3.3,

$$
P_{L}^{ \pm, c, h}\left(Q_{L}=\left\lfloor\theta c L^{d}\right\rfloor, M_{L}=\left\lfloor m L^{d}\right\rfloor\right)=\frac{K_{L}(m, \theta)}{Z_{L}}
$$

where

$$
K_{L}(m, \theta)=A_{L}^{\theta, c}(m) e^{h\left\lfloor m L^{d}\right\rfloor+\kappa\left\lfloor\theta c L^{d}\right\rfloor} \mathbb{P}_{L}^{ \pm, J}\left(M_{L}=\left\lfloor m L^{d}\right\rfloor\right) .
$$


Here $Z_{L}$ is the normalization constant from (3.6) which in the present formulation can also be interpreted as the sum of $K_{L}(m, \theta)$ over the relevant (discrete) values of $m$ and $\theta$.

Let $K_{L, \epsilon}(m, \theta)$ denote the sum of $K_{L}\left(m^{\prime}, \theta\right)$ over all $m^{\prime}$ and $\theta^{\prime}$ for which $m^{\prime} L^{d}$ and $\theta^{\prime} c L^{d}$ are integers and $\left|m^{\prime}-m\right| \leqslant \epsilon$ and $\left|\theta^{\prime} c-\theta c\right| \leqslant \epsilon$. (This is exactly the set of magnetizations and spin-salt overlaps contributing to the set $\mathcal{B}_{L, \epsilon}$.) Applying (3.1) to extract the exponential behavior of the last probability in (3.32), and using (3.9) to do the same for the quantity $A_{L}^{\theta, c}(m)$, we get

$$
\left|\frac{\log K_{L, \epsilon}(m, \theta)}{L^{d}}+\mathscr{G}_{h, c}(m, \theta)\right| \leqslant \epsilon+\epsilon^{\prime},
$$

where $\epsilon^{\prime}$ is as in (3.9). As a consequence of the above estimate we have

$$
\lim _{\epsilon \downarrow 0} \lim _{L \rightarrow \infty} \frac{\log K_{L, \epsilon}(m, \theta)}{L^{d}}=-\mathscr{G}_{h, c}(m, \theta)
$$

for any $m \in(-1,1)$ and any $\theta \in(0,1)$.

Next we will attend to the denominator in (3.31). Pick $\delta>0$ and consider the set

$$
\mathcal{M}_{\delta}=\{(m, \theta):|m| \leqslant 1-\delta, \delta \leqslant \theta \leqslant 1-\delta\} .
$$

We will write $Z_{L}$ as a sum of two terms, $Z_{L}=Z_{L}^{(1)}+Z_{L}^{(2)}$, with $Z_{L}^{(1)}$ obtained by summing $K(m, \theta)$ over the admissible $(m, \theta) \in \mathcal{M}_{\delta}$ and $Z_{L}^{(2)}$ collecting the remaining terms. By Lemma 3.6 we know that $Z_{L}^{(2)} / Z_{L}$ decays exponentially in $L^{d}$ and so the decisive contribution to $Z_{L}$ comes from $Z_{L}^{(1)}$. Assuming that $\epsilon \ll \delta$, let us cover $\mathcal{M}_{\delta}$ by finite number of sets of the form $\left[m_{\ell}^{\prime}-\epsilon, m_{\ell}^{\prime}+\epsilon\right] \times\left[\theta_{\ell}^{\prime}-\epsilon, \theta_{\ell}^{\prime}+\epsilon\right]$, where $m_{\ell}^{\prime}$ and $\theta_{\ell}^{\prime}$ are such that $m_{\ell}^{\prime} L^{d}$ and $\theta_{\ell}^{\prime} c L^{d}$ are integers. Then $Z_{L}^{(1)}$ can be bounded as in

$$
\max _{\ell} K_{L, \epsilon}\left(m_{\ell}^{\prime}, \theta_{\ell}^{\prime}\right) \leqslant Z_{L}^{(1)} \leqslant \sum_{\ell} K_{L, \epsilon}\left(m_{\ell}^{\prime}, \theta_{\ell}^{\prime}\right) .
$$

Moreover, the right-hand-side is bounded by the left-hand-side times a polynomial in $L$. Taking logarithms, dividing by $L^{d}$, taking the limit $L \rightarrow$ $\infty$, refining the cover and applying the continuity of $(m, \theta) \mapsto \mathscr{G}_{h, c}(m, \theta)$ allows us to conclude that

$$
\lim _{L \rightarrow \infty} \frac{\log Z_{L}}{L^{d}}=-\inf _{m \in(-1,1)} \inf _{\theta \in[0,1]} \mathscr{G}_{h, c}(m, \theta)
$$


Combining these observations, (2.8) is proved.

Proof of Theorem 2.1. The conclusion (2.8) follows from (3.30) by similar arguments that prove (3.37). The only remaining thing to prove is the strict convexity of $m \mapsto G_{h, c}(m)$ and continuity and monotonicity of its minimizer. First we note that $\theta \mapsto \mathscr{G}_{h, c}(m, \theta)$ is strictly convex on the set of $\theta$ where it is finite, which is a simple consequence of the strict convexity of $p \mapsto \mathscr{S}(p)$. Hence, for each $m$, there is a unique $\theta=\theta(m)$ which minimizes $\theta \mapsto \mathscr{G}_{h, c}(m, \theta)$.

Our next goal is to show that, for $\kappa c>0$, the solution $\theta=\theta(m)$ will satisfy the inequality

$$
\theta>\frac{1+m}{2}
$$

(A heuristic reason for this is that $\theta=\frac{1+m}{2}$ corresponds to the situation when the salt is distributed independently of the underlying spins. This is the dominating strategy for $\kappa=0$; once $\kappa>0$ it is clear that the fraction of salt on plus spins must increase.) A formal proof runs as follows: We first note that $m \mapsto \theta(m)$ solves for $\theta$ from the equation

$$
\frac{\partial}{\partial \theta} \Xi(m, \theta ; c)=-\kappa c
$$

where $\Xi(m, \theta ; c)$ is as in (2.7). But $\theta \mapsto \Xi(m, \theta ; c)$ is strictly concave and its derivative vanishes at $\theta=\frac{1}{2}(1+m)$. Therefore, for $\kappa c>0$ the solution $\theta=\theta(m)$ of (3.39) must obey (3.38).

Let $\mathcal{V}$ be the set of $(m, \theta) \in(-1,1) \times(0,1)$ for which (3.38) holds and note that $\mathcal{V}$ is convex. A standard second-derivative calculation now shows that $\mathscr{G}_{h, c}(m, \theta)$ is strictly convex on $\mathcal{V}$. (Here we actually differentiate the function $\mathscr{G}_{h, c}(m, \theta)-\mathscr{F}_{J}(m)$ - which is twice differentiable on the set where it is finite - and then use the known convexity of $\mathscr{F}_{J}(m)$. The strict convexity is violated on the line $\theta=\frac{1}{2}(1+m)$ where $(m, \theta) \mapsto \mathscr{G}_{h, c}(m, \theta)$ has a flat piece for $m \in\left[-m_{\star}, m_{\star}\right]$.) Now, since $\theta(m)$ minimizes $\mathscr{G}_{h, c}(m, \theta)$ for a given $m$, the strict convexity of $\mathscr{G}_{h, c}(m, \theta)$ on $\mathcal{V}$ implies that for any $\lambda \in$ $(0,1)$,

$$
\begin{aligned}
G_{h, c}\left(\lambda m_{1}+\right. & \left.(1-\lambda) m_{2}\right) \\
& \leqslant \mathscr{G}_{h, c}\left(\lambda m_{1}+(1-\lambda) m_{2}, \lambda \theta\left(m_{1}\right)+(1-\lambda) \theta\left(m_{2}\right)\right) \\
& <\lambda \mathscr{G}_{h, c}\left(m_{1}, \theta\left(m_{1}\right)\right)+(1-\lambda) \mathscr{G}_{h, c}\left(m_{2}, \theta\left(m_{2}\right)\right) \\
& =\lambda G_{h, c}\left(m_{1}\right)+(1-\lambda) G_{h, c}\left(m_{2}\right) .
\end{aligned}
$$


Hence, $m \mapsto G_{h, c}(m)$ is also strictly convex. The fact that $G^{\prime}(m)$ diverges as $m \rightarrow \pm 1$ is a consequence of the corresponding property of the function $m \mapsto \mathscr{F}_{J}(m)$ and the fact that the rest of $\mathscr{G}_{h, c}$ is convex in $m$.

As a consequence of strict convexity and the abovementioned "steepness" at the boundary of the interval $(-1,1)$, the function $m \mapsto G_{h, c}(m)$ has a unique minimizer for each $h \in \mathbb{R}$ and $c>0$, as long as the quantities from (3.13) satisfy $p_{ \pm}<1$. The minimizer is automatically continuous in $h$ and is manifestly non-decreasing. Furthermore, the continuity of $G_{h, c}$ in $c$ allows us to conclude that $\theta(\mathrm{m})$ is also continuous in $c$. What is left of the claims is the strict monotonicity of $m$ as a function of $h$. Writing $G_{h, c}(m)$ as $-h m+g(m)$ and noting that $g$ is continuously differentiable on $(-1,1)$, the minimizing $m$ satisfies

$$
g^{\prime}(m)=h .
$$

But $g(m)$ is also strictly convex and so $g^{\prime}(m)$ is strictly increasing. It follows that $m$ has to be strictly increasing with $h$.

Theorem 3.1 has the following simple consequence that is worth highlighting:

Corollary 3.8. For given $h \in \mathbb{R}$ and $c \in(0,1)$, let $(m, \theta)$ be the minimizer of $\mathscr{G}_{h, c}(m, \theta)$. Then for all $\epsilon>0$,

$$
\lim _{L \rightarrow \infty} P_{L}^{ \pm, c, h}\left(\left|Q_{L}-\theta c L^{d}\right| \geqslant \epsilon L^{d} \text { or }\left|M_{L}-m L^{d}\right| \geqslant \epsilon L^{d}\right)=0 .
$$

Proof. On the basis of (3.30) and the fact that $\mathscr{G}_{h, c}(m, \theta)$ has a unique minimizer, a covering argument - same as used to prove (3.37) implies that the probability on the left-hand-side decays to zero exponentially in $L^{d}$.

Before we proceed to the proof of our second main theorem, let us make an observation concerning the values of $p_{ \pm}$at the minimizing $m$ and $\theta$ :

Lemma 3.9. Let $h \in \mathbb{R}$ and $c \in(0,1)$ be fixed and let $(m, \theta)$ be the minimizer of $\mathscr{G}_{h, c}(m, \theta)$. Define the quantities $q_{ \pm}=q_{ \pm}(m, c, \kappa)$ by $(2.14)$ and $p_{ \pm}=p_{ \pm}(m, \theta, c)$ by $(3.13)$. Then

$$
q_{+}=p_{+} \quad \text { and } \quad q_{-}=p_{-} .
$$

Moreover, $q_{ \pm}$are then related to $h$ via (2.17) whenever $m \in\left[-m_{\star}, m_{\star}\right]$. 
Proof. First let us ascertain that $q_{ \pm}$are well defined from equations (2.14). We begin by noting that the set of possible values of $\left(q_{+}, q_{-}\right)$ is the unit square $[0,1]^{2}$. As is easily shown, the first equation in (2.14) corresponds to an increasing curve in $[0,1]^{2}$ connecting the corners $(0,0)$ and $(1,1)$. On the other hand, the second equation in (2.14) is a straight line with negative slope which by the fact that $c<1$ intersects both the top and the right side of the square. It follows that these curves intersect at a single point - the unique solution of (2.14).

Next we will derive equations that $p_{ \pm}$have to satisfy. Let $(m, \theta)$ be the unique minimizer of $\mathscr{G}_{h, c}(m, \theta)$. The partial derivative with respect to $\theta$ yields

$$
c\left(\mathscr{S}^{\prime}\left(p_{+}\right)-\mathscr{S}^{\prime}\left(p_{-}\right)\right)=\kappa c
$$

and from the very definition of $p_{ \pm}$we have

$$
\frac{1+m}{2} p_{+}+\frac{1-m}{2} p_{-}=c
$$

Noting that $\mathscr{S}^{\prime}(p)=\log \frac{p}{1-p}$, we now see that $p_{ \pm}$satisfies the same equations as $q_{ \pm}$and so, by the above uniqueness argument, (3.43) must hold.

To prove relation (2.17), let us also consider the derivative of $\mathscr{G}_{h, c}(m, \theta)$ with respect to $m$. For solutions in $\left[-m_{\star}, m_{\star}\right]$ we can disregard the $\mathscr{F}_{J}$ part of the function (because its vanishes along with its derivative throughout this interval), so we have

$$
h=-\frac{\partial}{\partial m} \Xi(m, \theta ; c) .
$$

A straightforward calculation then yields (2.17).

Now we are ready to prove our second main result:

Proof of Theorem 2.2. The crucial technical step for the present proof has already been established in Lemma 3.3. In order to plug into the latter result, let us note that the sum of $e^{\kappa Q_{L}(\sigma, S)}$ over all salt configurations $S=\left(S_{x}\right) \in\{0,1\}^{\Lambda_{L}}$ with $N_{L}=\left\lfloor c L^{d}\right\rfloor$ is a number depending only on the total magnetization $M_{L}=M_{L}(\sigma)$. Lemma 3.3 then implies

$$
\begin{aligned}
P_{L}^{ \pm, c, h}\left(\mathcal{A} \times\{0,1\}^{\Lambda_{L}} \cap\left\{M_{L}\right.\right. & \left.\left.=\left\lfloor m L^{d}\right\rfloor\right\}\right) \\
& =\omega_{L}(m) \mathbb{P}_{L}^{ \pm, J}\left(\mathcal{A} \cap\left\{M_{L}=\left\lfloor m L^{d}\right\rfloor\right\}\right)
\end{aligned}
$$


where $\omega_{L}(m)$ is a positive number depending on $m$, the parameters $c, h, J$ and the boundary condition \pm but not on the event $\mathcal{A}$. Noting that $\rho_{L}^{ \pm}$is simply the distribution of the random variables $M_{L} / L^{d}$ in measure $P_{L}^{ \pm, c, h}$, this proves (2.12).

In order to prove the assertion (2.13), we let $\bar{\sigma} \in\{0,1\}^{\Lambda_{L}}$, pick $\Lambda \subset \Lambda_{L}$ and fix $\bar{S} \in\{0,1\}^{\Lambda}$. Since Lemma 3.3 guarantees that, given $\{\sigma=\bar{\sigma}\}$, all salt configurations with fixed $Q_{L}$ and concentration $c$ have the same probability in $P_{L}^{ \pm, c, h}(\cdot \mid \sigma=\bar{\sigma})$, we have

$$
P_{L}^{ \pm, c, h}\left(S_{\Lambda}=\bar{S}_{\Lambda}, S \in \mathcal{A}_{L}^{\theta, c}(\bar{\sigma}) \mid \sigma=\bar{\sigma}\right)=R_{\Lambda, L}^{\theta, c}\left(\bar{\sigma}, S_{\Lambda}\right),
$$

where $R_{\Lambda, L}^{\theta, c}$ is defined in (3.12). Pick $\eta>0$ and assume, as in Lemma 3.5, that $c \in[\eta, 1-\eta], \theta \in[\eta, 1-\eta]$ and $M_{L}(\bar{\sigma})=\left\lfloor m L^{d}\right\rfloor$ for some $m$ with $|m| \leqslant$ $1-\eta$. Then the aforementioned lemma tells us that $R_{\Lambda, L}^{\theta, c}(\bar{\sigma}, \cdot)$ is within $\epsilon$ of the probability that $\bar{S}_{\Lambda}$ occurs in the product measure where the probability of $S_{x}=1$ is $p_{+}$if $\bar{\sigma}_{x}=+1$ and $p_{-}$if $\bar{\sigma}_{x}=-1$.

Let $(m, \theta)$ be the unique minimizer of $\mathscr{G}_{h, c}(m, \theta)$. Taking expectation of (3.48) over $\bar{\sigma}$ with $\bar{\sigma}_{\Lambda}$ fixed, using Corollary 3.8 to discard the events $\left|M_{L} / L^{d}-m\right| \geqslant \epsilon$ or $\left|Q_{L} / L^{d}-\theta c\right| \geqslant \epsilon$ and invoking the continuity of $p_{ \pm}$in $m$ and $\theta$, we find out that $P_{L}^{ \pm, c, h}\left(S_{\Lambda}=\bar{S}_{\Lambda} \mid \sigma_{\Lambda}=\bar{\sigma}_{\Lambda}\right)$ indeed converges to

$$
\prod_{x \in \Lambda}\left\{p_{\bar{\sigma}_{x}} \delta_{1}\left(\bar{S}_{x}\right)+\left(1-p_{\bar{\sigma}_{x}}\right) \delta_{0}\left(\bar{S}_{x}\right)\right\}
$$

with $p_{ \pm}$evaluated at the minimizing $(m, \theta)$. But for this choice Lemma 3.9 guarantees that $p_{ \pm}=q_{ \pm}$, which finally proves (2.13-2.14).

The last item to be proved is Proposition 2.4 establishing the basic features of the phase diagram of the model under consideration:

Proof of Proposition 2.4. From Lemma 3.9 we already know that the set of points $m(h, c)=m$ for $m \in\left[-m_{\star}, m_{\star}\right]$ is given by the Eq. (2.17). By the fact that $m(h, c)$ is strictly increasing in $h$ and that $m(h, c) \rightarrow \pm 1$ as $h \rightarrow \pm \infty$ we thus know that (2.17) defines a line in the $(h, c)$-plane. Specializing to $m= \pm m_{\star}$ gives us two curves parametrized by functions $c \mapsto$ $h_{ \pm}(c)$ such that at $(h, c)$ satisfying $h_{-}(c)<h<h_{+}(c)$ the system magnetization $m(h, c)$ is strictly between $-m_{\star}$ and $m_{\star}$, i.e., $(h, c)$ is in the phase separation region.

It remains to show that the above functions $c \mapsto h_{ \pm}(c)$ are strictly monotone and negative for $c>0$. We will invoke the expression (2.17) 
which applies because on the above curves we have $m(h, c) \in\left[-m_{\star}, m_{\star}\right]$. Let us introduce new variables

$$
R_{+}=\frac{q_{+}}{1-q_{+}} \quad \text { and } \quad R_{-}=\frac{q_{-}}{1-q_{-}}
$$

and, writing $h$ in (2.17) in terms of $R_{ \pm}$, let us differentiate with respect to $c$. (We will denote the corresponding derivatives by superscript prime.) Since (2.14) gives us that $R_{-}=e^{-\kappa} R_{+}$, we easily derive

$$
2 h^{\prime}=\frac{R_{-}^{\prime}}{1+R_{-}}-\frac{R_{+}^{\prime}}{1+R_{+}}=-R_{+}^{\prime} \frac{1-e^{-\kappa}}{\left(1+R_{+}\right)\left(1+R_{-}\right)} .
$$

Thus, $h^{\prime}$ and $R_{+}^{\prime}$ have opposite signs; i.e., we want to prove that $R_{+}^{\prime}>0$. But that is immediate: By the second equation in (2.14) we conclude that at least one of $R_{ \pm}^{\prime}$ must be strictly positive, and by $R_{-}=e^{-\kappa} R_{+}$we find that both $R_{ \pm}^{\prime}>0$. It follows that $c \mapsto h_{ \pm}(c)$ are strictly decreasing, and since $h_{ \pm}(0)=0$, they are also negative once $c>0$.

\section{ACKNOWLEDGMENTS}

The research of K.S.A. was supported by the NSF under the grants DMS-0103790 and DMS-0405915. The research of M.B. and L.C. was supported by the NSF grant DMS-0306167.

\section{REFERENCES}

1. K. S. Alexander, M. Biskup, and L. Chayes, Colligative properties of solutions II. Vanishing concentrations, next paper.

2. K. Alexander, J. T. Chayes, and L. Chayes, The Wulff construction and asymptotics of the finite cluster distribution for two-dimensional Bernoulli percolation, Commun. Math. Phys. 131:1-51, (1990).

3. Ph. Blanchard, L. Chayes, and D. Gandolfo, The random cluster representation for the infinite-spin Ising model: Application to QCD pure gauge theory, Nucl. Phys. B [FS] 588:229-252 (2000) .

4. M. Biskup, L. Chayes, and R. Kotecký, Critical region for droplet formation in the two-dimensional Ising model, Commun. Math. Phys. 242(1-2):137-183 (2003).

5. M. Biskup, L. Chayes, and R. Kotecký, Comment on: "Theory of the evaporation/condensation transition of equilibrium droplets in finite volumes", Physica A 327:589-592 (2003).

6. T. Bodineau, The Wulff construction in three and more dimensions, Commun. Math. Phys. 207:197-229 (1999).

7. T. Bodineau, Slab Percolation for the Ising Model, math.PR/0309300.

8. T. Bodineau, D. Ioffe and Y. Velenik, Rigorous probabilistic analysis of equilibrium crystal shapes, J. Math. Phys. 41:1033-1098 (2000). 
9. R. Cerf, Large deviations for three dimensional supercritical percolation, Astérisque 267:vi+177 (2000).

10. R. Cerf and A. Pisztora, On the Wulff crystal in the Ising model, Ann. Probab. 28:9471017 (2000).

11. A. H. Cottrell, Theoretical Structural Metallurgy, (St. Martin's Press, New York, 1955).

12. P. Curie, Sur la formation des cristaux et sur les constantes capillaires de leurs différentes faces, Bull. Soc. Fr. Mineral. 8:145 (1885); Reprinted in CEuvres de Pierre Curie, Gauthier-Villars, Paris, 1908, pp. 153-157.

13. A. Dembo and O. Zeitouni, Large Deviations Techniques and Applications (Springer Verlag, Inc., New York, 1998).

14. R. L. Dobrushin, R. Kotecký and S. B. Shlosman, Wulff construction. A Global Shape from Local Interaction, (Amer. Math. Soc., Providence, RI, 1992).

15. R. L. Dobrushin and S. B. Shlosman, in Probability Contributions to Statistical Mechanics, (Amer. Math. Soc., Providence, RI, 1994), pp. 91-219.

16. J. W. Gibbs, On the equilibrium of heterogeneous substances (1876), in Collected Works, Vol. 1., (Longmans, Green and Co., 1928).

17. K. M. Golden, Critical behavior of transport in sea ice, Physica B 338:274-283 (2003).

18. K. M. Golden, S. F. Ackley, and V. I. Lytle, The percolation phase transition in sea ice, Science 282:2238-2241 (1998).

19. P. E. Greenwood and J. Sun, Equivalences of the large deviation principle for Gibbs measures and critical balance in the Ising model, J. Statist. Phys. 86(1-2):149-164 (1997).

20. P. E. Greenwood and J. Sun, On criticality for competing influences of boundary and external field in the Ising model, J. Statist. Phys. 92(1-2):35-45 (1998).

21. F. den Hollander, Large Deviations, Fields Institute Monographs, Vol 14 (American Mathematical Society, Providence, RI, 2000).

22. D. Ioffe and R. H. Schonmann, Dobrushin-Kotecký-Shlosman theorem up to the critical temperature, Commun. Math. Phys. 199:117-167 (1998).

23. R. Kotecký and I. Medved', Finite-size scaling for the 2D Ising model with minus boundary conditions, J. Statist. Phys. 104(5/6):905-943 (2001).

24. L. D. Landau and E. M. Lifshitz, Statistical Physics, Course of Theoretical Physics, Vol. 5 (Pergamon Press, New York, 1977).

25. T. D. Lee and C. N. Yang, Statistical theory of equations of state and phase transitions: II. Lattice gas and Ising model, Phys. Rev. 87:410-419 (1952).

26. M. Matsumoto, S. Saito and I. Ohmine, Molecular dynamics simulation of the ice nucleation and proces leading to water freezing, Nature 416:409-413 (2002).

27. W. J. Moore, Physical Chemistry, 4th ed. (Prentice Hall, Inc., Englewood Cliffs, NJ 1972).

28. C.-E. Pfister, Large deviations and phase separation in the two-dimensional Ising model, Helv. Phys. Acta 64:953-1054 (1991).

29. C.-E. Pfister and Y. Velenik, Large deviations and continuum limit in the 2D Ising model, Probab. Theory Rel. Fields 109:435-506 (1997).

30. R. H. Schonmann and S. B. Shlosman, Constrained variational problem with applications to the Ising model, J. Statist. Phys. 83(5-6):867-905 (1996).

31. Ya. G. Sinaĩ, Theory of Phase Transitions: Rigorous Results, International Series in Natural Philosophy, Vol. 108 (Pergamon Press, Oxford-Elmsford, NY, 1982).

32. C. Rottman and M. Wortis, Statistical mechanics of equilibrium crystal shapes: Interfacial phase diagrams and phase transitions, Phys. Rep. 103:59-79 (1984).

33. G. Wulff, Zur Frage des Geschwindigkeit des Wachsturms und der Auflösung der Krystallflachen, Z. Krystallog. Mineral. 34:449-530 (1901). 